\title{
Harmonic functions on metric graphs under the anti-Kirchhoff law
}

\author{
Joachim von Below* and José A. Lubary ${ }^{\dagger}$
}

\begin{abstract}
When does an infinite metric graph allow nonconstant bounded harmonic functions under the anti-Kirchhoff transition law? We give a complete answer to this question in the cases where Liouville's Theorem holds, for trees, for graphs with finitely many essential ramification nodes and for generalized lattices. It turns out that the occurrence of nonconstant bounded harmonic functions under the anti-Kirchhoff law differs strongly from the one under the classical continuity and the Kirchhoff flow condition.
\end{abstract}

Keywords: harmonic functions, Liouville's Theorem, infinite graphs, metric graphs, quantum graphs, anti-Kirchhoff law, generalized lattices.

MSC (2010): 35R02, 35J25, 34B45, 05C50, 05C10, 05C63, 31C05.

Among the classical and often considered transition conditions at the nodes of a metric graph we find the continuity condition at the ramification nodes

$$
\forall v_{i} \in V_{\mathrm{r}}: k_{j} \cap k_{s}=\left\{v_{i}\right\} \Longrightarrow u_{j}\left(v_{i}\right)=u_{s}\left(v_{i}\right) \text {, }
$$

and Kirchhoff's flow law, by which, at each node $v_{i}$, all the incident outer normal derivatives sum up to 0 :

$$
\sum_{j} d_{i j} \partial_{j} u_{j}\left(v_{i}\right)=0
$$

We shall cite both conditions (1) and (2) together as $(C K)$. They have been treated by many authors, including generalizations as weighted Kirchhoff conditions and dynamical ones in connection with differential operators on the edges, and are of interest in many settings and applications.

However, when treating wave dispersion problems on graphs e.g., or as in many other cases, the $(C K)$-condition is not suitable and should be replaced by its orthogonal condition, the so-called anti-Kirchhoff condition $(K C)$, see [6] and the

\footnotetext{
${ }^{*}$ corresponding author

†Supported by MINECO grants MTM2014-52402-C3-1-P and MTM2017-84214-C2-1-P and part of the Catalan research group 2017 SGR 1392.
} 
references therein. It is given by the continuity of the outer normal derivatives at the ramification nodes (4) and by vanishing potential sums at all vertices (3). Mathematically it stems from the self-adjoint orthogonal boundary condition in the sense of the $Y$-boundary conditions associated to corresponding Bochner-spaces, see [6].

The present paper deals with the validity of Liouville's Theorem on infinite uniformly locally finite metric graphs under the anti-Kirchhoff law, or more generally, with the multiplicity of 0 as an eigenvalue of the edge Laplacian under $(K C)$ in $L^{\infty}(G)$. It turns out that the $(K C)$-condition enforces a behaviour of bounded harmonic functions that differs strongly from the one under the $(C K)$-condition. Denoting the edges of the graph by $k_{j}$, at each vertex $v_{i}$ we impose the following transition condition

$$
\begin{gathered}
\sum_{v_{i} \in k_{j}} u_{j}\left(v_{i}\right)=0, \\
k_{j} \cap k_{s}=\left\{v_{i}\right\} \Longrightarrow d_{i j} \partial_{j} u_{j}\left(v_{i}\right)=d_{i s} \partial_{s} u_{s}\left(v_{i}\right) .
\end{gathered}
$$

Conceivably, we shall cite both conditions (3) and (4) together as $(K C)$. Note that (3) reduces to the 0 -Dirichlet condition at boundary vertices.

The presentation is organized as follows. After some prerequisites, graph theoretical preliminaries and basic facts about harmonic functions in Section 1, Section 2 deals with the finite case. It turns out that a finite metric graph $\Gamma$ under $(K C)$ is a Liouville graph if and only if $\Gamma$ is a tree or a non bipartite unicyclic graph. Section 3 presents some basic tools for the infinite case, especially the distinctive impact of circuits on the occurrence of nonconstant bounded harmonic functions. These results lead among others to the characterization of infinite Liouville graphs and of trees without two-sided unbounded paths in Section 4. In particular it will be shown that a graph $\Gamma$ containing an one-sided unbounded path $\Gamma_{0}$ of infinite total length is a Liouville graph under $(K C)$ if and only if it is a sole $\Gamma_{0}$ with finite trees attached to its vertices, see Theorem 4.1 .

In Sections 5 and 6 the occurrence of bounded harmonic functions on infinite graphs with finitely many essential ramification nodes, the so-called medusae, and infinite trees will be treated. In the first case, optimal upper and lower bounds for the dimension $m(0 ; \Gamma ; K C)$ of the vector space of bounded harmonic functions under $(K C)$ in dependence of the smallest connected finite graph containing the essential ramification nodes will be established, see Theorems 5.1 and 5.2. As for infinite trees it will be shown that a tree $T$ satisfies $m(0 ; \Gamma ; K C)=\infty$ if and only if its reduced tree has infinitely many essential ramification nodes, see Theorem 6.2. In contrast to the $(C K)$-condition, no geometrical restriction to the edges is necessary here.

In Section 7 graphs with exactly one independent bounded harmonic function and generalized lattices, the so-called periodic graphs will be treated. It turns out that all periodic graphs containing circuits fulfill $m(0 ; \Gamma ; K C)=\infty$, even with inseparable eigenspaces, while periodic trees are precisely those periodic graphs fulfilling $m(0 ; \Gamma ; K C)=1$. Here, the behaviour under $(K C)$ is in total contrast to the one under $(C K)$ where every periodic graph is a Liouville graph.

A final section containing remarks and supplements is added. 


\section{Metric graphs and harmonic functions}

For any graph $\Gamma=(V, E, \in)$, the vertex set is denoted by $V=V(\Gamma)$, the edge set by $E=E(\Gamma)$ and the incidence relation by $\in \subset V \times E$. The valency or degree of each vertex $v$ is denoted by

$$
\gamma(v)=\gamma(v ; \Gamma)=\#\{k \in E(\Gamma) \mid v \in k\} .
$$

The adjacency of two vertices $v, w \in V(\Gamma)$ will be denoted by $v \sim w$.

Unless otherwise stated, all graphs considered in this paper are assumed to be nonempty, countable, connected and uniformly locally finite. The last property means that

$$
\max _{v \in V(\Gamma)} \gamma(v)=: \gamma_{\max }<\infty .
$$

Accordingly, the vertices will be numbered by $v_{i}$ with $i \in \mathcal{I} \subset \mathbb{N}$, the respective valencies by $\gamma_{i}=\gamma\left(v_{i}\right)$, and the edges by $k_{j}$ with $j \in \mathcal{J} \subset \mathbb{N}$. The boundary vertices $V_{\mathrm{b}}=\{v \in V(\Gamma) \mid \gamma(v)=1\}$ will be distinguished from the ramification nodes $V_{\mathrm{r}}=\{v \in V(\Gamma) \mid \gamma(v) \geq 2\}$ and the essential ramification nodes $V_{\mathrm{ess}}=\{v \in$ $V(\Gamma) \mid \gamma(v) \geq 3\}$. By definition, a circuit is a connected and regular graph of valency 2. This includes the infinite circuit in the form of the two-sided unbounded path $\Gamma_{1}$. A finite path is a connected graph with two distinct vertices of valency 1 while the other vertices are all of valency 2 .

The sequences or column vectors with constant entries equal to 1 are denoted by $\mathbf{e}$ and $\mathbf{e}^{n}$ if the dimension $n$ is to be noted. The $n$-vectors of the canonical basis are denoted by $\mathbf{e}_{k}^{n}=\left(\delta_{i k}\right)_{n \times 1}$. For a subgraph $\Theta \leq \Gamma$ let $\bar{\Theta}=(V(\Theta), E(\bar{\Theta}), \in)$ denote the subgraph of $\Gamma$ spanned by the vertices in $\Theta$ with

$$
E(\bar{\Theta})=\{e \mid e \in E(\Gamma), e \cap V(\Gamma) \subset V(\Theta)\} .
$$

The subgraph $\Theta$ is called induced if $\bar{\Theta}=\Theta$, i.e. $\Theta$ contains all edges in $\Gamma$ that have their vertices in $\Theta$. Two subgraphs are called vertex independent if they have no vertex in common, and essentially or almost disjoint if they have only a finite number of vertices in common. For further graph theoretical terminology we refer to $[7,11]$.

Moreover, we consider each graph as a connected topological graph in $\mathbb{R}^{m}$, i.e. $V(\Gamma) \subset \mathbb{R}^{m}$ and the edge set consists in a collection of Jordan curves

$$
E(\Gamma)=\left\{\pi_{j}:\left[0, \ell_{j}\right] \rightarrow \mathbb{R}^{m} \mid j \in \mathcal{J}\right\}
$$

with the following properties: Each support $k_{j}:=\pi_{j}\left(\left[0, \ell_{j}\right]\right)$ has its endpoints in the set $V(\Gamma)$, any two vertices in $V(\Gamma)$ can be connected by a path with arcs in $E(\Gamma)$, and any two edges $k_{j} \neq k_{h}$ satisfy $k_{j} \cap k_{h} \subset V(\Gamma)$ and $\#\left(k_{j} \cap k_{h}\right) \leq 1$. The arc length parameter of an edge $k_{j}$ is denoted by $x_{j}$. Unless otherwise stated, we identify the graph $\Gamma=(V, E, \in)$ with its associated metric graph, network or quantum graph

$$
G=\bigcup_{j \in \mathcal{J}} \pi_{j}\left(\left[0, \ell_{j}\right]\right),
$$


especially each edge $\pi_{j}$ with its support $k_{j}$. Throughout it will be assumed that all $\pi_{j}$ are at least Lipschitz continuous. Thus, endowed with the induced topology $G$ is a connected space in $\mathbb{R}^{m}$. Throughout, we shall denote the total graph length by

$$
L(\Gamma)=L(G)=\sum_{j \in \mathcal{J}} \ell_{j}
$$

The orientation of the graph $\Gamma$ is given by the incidence matrix $\mathcal{D}(\Gamma)=\left(d_{i k}\right)_{\mathcal{I} \times \mathcal{J}}$ with

$$
d_{i j}= \begin{cases}1 & \text { if } \pi_{j}\left(\ell_{j}\right)=v_{i} \\ -1 & \text { if } \pi_{j}(0)=v_{i} \\ 0 & \text { otherwise }\end{cases}
$$

This allows a refinement of the valency notion by defining the outdegree $\gamma^{-}(v)$ and indegree $\gamma^{+}(v)$ of a vertex $v$ by

$$
\begin{aligned}
& \gamma^{-}(v ; \Gamma)=\#\left\{k_{j} \in E(\Gamma) \mid d_{i j}=-1\right\} \\
& \gamma^{+}(v ; \Gamma)=\#\left\{k_{j} \in E(\Gamma) \mid d_{i j}=1\right\}
\end{aligned}
$$

The corank of the graph $\Gamma$ is defined by

$$
\operatorname{corank}(\Gamma)=\operatorname{dim} \operatorname{ker} \mathcal{D}(\Gamma)
$$

that in the finite case amounts to $\# E(\Gamma)-\# V(\Gamma)+1$ and, thereby, reduces to the value 1 if and only if $\Gamma$ is unicyclic. In general, the circuit space being defined as $\Pi(\Gamma)=\langle c \in \operatorname{ker} \mathcal{D}(\Gamma) \mid \# \operatorname{supp}(c)<\infty\rangle$ is only a subspace of $\operatorname{ker} \mathcal{D}(\Gamma)$ and not identical with it. However, it holds

$$
\operatorname{dim} \Pi(\Gamma)=\operatorname{dim} \operatorname{ker} \mathcal{D}(\Gamma)
$$

see e.g. [4]. In the context of the anti-Kirchhoff law, the generalized unsigned circuit space defined by

$$
\Pi^{+}(\Gamma)=\left\{c \in \mathbb{R}^{E(\Gamma)} \mid \forall v_{i} \in V(\Gamma): \sum_{j \in \mathcal{J}} d_{i j}^{2} c_{j}=0\right\}
$$

plays a crucial role and is in fact just the kernel of the unsigned incidence operator given by the matrix $\left(\left|d_{i j}\right|\right)_{\mathcal{I} \times \mathcal{J}}$. In particular in the finite not necessarily connected case, we note that

$$
\operatorname{dim} \Pi^{+}(\Gamma)=\# E(\Gamma)-\# V(\Gamma)+c^{+}(\Gamma)
$$

where $c^{+}(\Gamma)$ stands for the number of bipartite connected components of $\Gamma$.

For vectors of functions $u:=\left(u_{j}\right)_{j \in \mathcal{J}}$ defined by their edge components $u_{j}$ : $\left[0, \ell_{j}\right] \rightarrow \mathbb{C}$ we use the abbreviations

$$
u_{j}\left(v_{i}\right):=u_{j}\left(\pi_{j}^{-1}\left(v_{i}\right)\right), \quad \partial_{j} u_{j}\left(v_{i}\right):=\left.\frac{\partial}{\partial x_{j}} u_{j}\left(x_{j}\right)\right|_{\pi_{j}^{-1}\left(v_{i}\right)} \text { etc. }
$$


Endowed with a usual product norm we introduce for $k \in \mathbb{N}$ and $p \in[1, \infty]$

$$
\mathcal{V}^{k}(G)=\prod_{j \in \mathcal{J}} \mathcal{C}^{k}\left[0, \ell_{j}\right] \quad L^{p}(G)=\prod_{j \in \mathcal{J}} L^{p}\left(0, \ell_{j}\right)
$$

The validity of the anti-Kirchhoff conditions (3) and (4) in a function space will be indicated by the subscript $(K C)$, the one of $(C K)$ by the subscript $C K$ correspondingly.

Of course, harmonic functions $u:=\left(u_{j}\right)_{j \in \mathcal{J}}$ on a metric graph $G$ are defined as solutions of $\Delta u=0$ under suitable transition conditions. But, as these solutions are affine linear on each edge, we can define a harmonic function intrinsically without higher regularity assumptions on the arc length parametrizations than the rectifiability condition. Conceivably, a function $u:=\left(u_{j}\right)_{j \in \mathcal{J}}$ with $u_{j} \in \mathcal{C}\left[0, \ell_{j}\right]$ is called harmonic if on each edge $e_{j}$ it is of the form $u_{j}\left(x_{j}\right)=u_{j}(0)+\alpha_{j} x_{j}$ and satisfies the anti-Kirchhoff condition $(K C)$. The vector space of all harmonic functions on $G$ satisfying $(K C)$ will be denoted by $\mathcal{H}_{K C}(G)$. Correspondingly, we shall write $\mathcal{H}_{C K}(G)$ etc. Moreover, set

$$
\begin{aligned}
& \mathcal{H}_{K C}^{\infty}(G)=\mathcal{H}_{K C}(G) \cap L^{\infty}(G), \\
& \Pi_{\infty}^{+}(\Gamma)=\Pi^{+}(\Gamma) \cap \ell^{\infty}(E(\Gamma)) .
\end{aligned}
$$

Definition $1.1 \quad m(0 ; \Gamma ; K C)=\operatorname{dim} \mathcal{H}_{K C}^{\infty}(G)$.

If $G$ is a $\mathcal{C}^{2}$-metric graph, then clearly

$$
m(0 ; \Gamma ; K C)=m_{a}\left(0 ; \Delta^{K C} ; \mathcal{V}^{2}(G) \cap L^{\infty}(G)\right)=\operatorname{dim} E_{0}\left(\Delta^{K C} ; \mathcal{V}^{2}(G) \cap L^{\infty}(G)\right) \text {. }
$$

Definition 1.2 A metric graph $G$ is called a Liouville graph under $(K C)$, if each harmonic bounded function on $G$ fulfilling (3) and (4) is constant, i.e. if

$$
m(0 ; \Gamma ; K C)=0 .^{*}
$$

Unless otherwise stated, the harmonic functions to be considered will be supposed to fulfill $(K C)$.

Note that any $u=\left(u_{j}\right)_{j \in \mathcal{J}} \in \mathcal{H}_{K C}(G)$ has edge restrictions of the form $u_{j}\left(x_{j}\right)=$ $u_{j}(0)+\alpha_{j} x_{j}$ with slopes

$$
\alpha_{j}=\frac{u_{j}\left(\ell_{j}\right)-u_{j}(0)}{\ell_{j}}=\partial_{j} u_{j}\left(\pi_{j}\left(\ell_{j}\right)\right) .
$$

Moreover, by (4), at each vertex $v_{i}$ all incident normal derivatives $d_{i j} \partial_{j} u_{j}\left(v_{i}\right)$ coincide. Conceivably, we can set

$$
\nu\left(v_{i}\right)=\nu_{i}=d_{i j} \partial_{j} u_{j}\left(v_{i}\right)
$$

*In order to be consistent with Liouville's Theorem in Riemannian or complex manifolds, the authors prefer the present definition of the Liouville property instead of defining it by the multiplicity being equal to 1 as in the $(C K)$-case. In the latter one this is conceivable, since all constant functions are solutions. But, under $(K C)$, the only constant solution is the trivial one and, thereby, the solution characterizing the Liouville property. 
for some incident edge $k_{j}$ with $v_{i}$. Thus, by connectedness, there is a constant slope factor $\nu=\nu(u)$ such that

$$
\forall i \in \mathcal{I}:\left|\nu_{i}\right|=|\nu|
$$

and

$$
v_{i} \sim v_{h} \Rightarrow \nu_{h}=-\nu_{i}
$$

This yields immediately the

Corollary 1.3 If $\Gamma$ is not bipartite, then $\Pi_{\infty}^{+}(\Gamma) \cong \mathcal{H}_{K C}^{\infty}(G)$.

But in the bipartite case, there can be bounded harmonic functions with nonvanishing slope factor $\nu$, see Sections 5 and 6 . Of course, (7) does not mean that $m(0 ; \Gamma ; K C) \leq \operatorname{dim} \Pi_{\infty}^{+}+1$, see Section 5 .

\section{The finite case}

Suppose $\Gamma$ is a finite connected graph with $n$ vertices and $N$ edges. In the case of all edge lengths equal to 1 , it has been shown in [6] that 0 is not an eigenvalue of $\Delta^{K C}$ on trees or on non bipartite unicyclic graphs, since its algebraic multiplicity amounts to $N-n+1$ or to $N-n$, respectively. The proof given there is readily extended to arbitrary edge lengths. First, we note that a harmonic function under $(K C)$ on $\Gamma$ is constant on each edge, i.e. $\nu=0$, since

$$
\begin{aligned}
0=\sum_{j=1}^{N} \int_{0}^{\ell_{j}}\left(\partial_{j}^{2} u_{j}\right) u_{j} d x_{j} & =-\sum_{j=1}^{N} \int_{0}^{\ell_{j}}\left(\partial_{j} u_{j}\right)^{2} d x_{j}+\sum_{i=1}^{n} \nu_{i} \sum_{v_{i} \in k_{j}} u_{j}\left(v_{i}\right) \\
& =-\sum_{j=1}^{N} \int_{0}^{\ell_{j}}\left(\partial_{j} u_{j}\right)^{2} d x_{j} .
\end{aligned}
$$

Thus, the eigenspace belonging to 0 of the Laplacian under $(K C)$ satisfies

$$
E_{0}\left(\Gamma ; \Delta^{K C}\right) \cong \Pi^{+}(\Gamma) \text {. }
$$

Introduce

$$
\mathcal{M}(\Gamma)=\left\{M \mid M=\left(m_{i h}\right)_{n \times n}, \forall i, h \in\{1, \ldots, n\}: v_{i} \not v_{h} \Rightarrow m_{i h}=0\right\}
$$

and

$$
\mathcal{M}^{+}(\Gamma)=\left\{M \in \mathcal{M}(\Gamma) \mid M^{*}=M, M \mathbf{e}=0\right\} .
$$

The Hadamard multiplication with the length adjacency matrix is an isomorphism of $\mathcal{M}(\Gamma)$, while the dimension of $\mathcal{M}^{+}(\Gamma)$ has been determined in [1, Section 5]. This leads to the following result.

Lemma 2.1 If $\Gamma$ is a finite connected graph, then

$$
m(0 ; \Gamma ; K C)=\operatorname{dim} \mathcal{M}^{+}(\Gamma)= \begin{cases}N-n+1 & \text { if } \Gamma \text { is bipartite, } \\ N-n & \text { if } \Gamma \text { is not bipartite. }\end{cases}
$$

In particular, $\Gamma$ is a Liouville graph under $(K C)$ if and only if $\Gamma$ is a tree or a non bipartite unicyclic graph. 


\section{Basic facts}

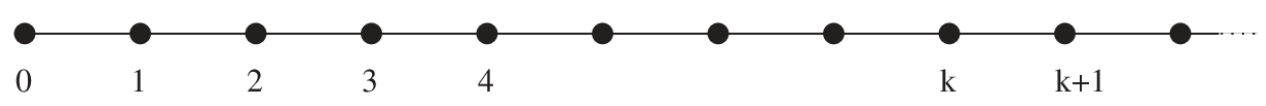

Figure 1: The one-sided unbounded path $\Gamma_{0}$.

First, we consider the smallest infinite graph.

Example 3.1 The one-sided unbounded path $\Gamma_{0}$ consists in the vertex set $\mathbb{N}$ with edges defined by the natural incidences

$$
d_{i i}=-1 \quad \text { and } \quad d_{i+1, i}=1
$$

Clearly, $\Pi_{\infty}^{+}\left(\Gamma_{0}\right)=\{0\}$. Suppose $u \in \mathcal{H}_{K C}^{\infty}\left(\Gamma_{0}\right)$ satisfies $u_{0}^{\prime}(0)=\nu$, and $u_{0}(0)=0$. Set $y_{k}=u_{k}(0)$ and get recursively

$$
\forall k \in \mathbb{N}: y_{k+1}=-\left(y_{k}+(-1)^{k} \nu \ell_{k}\right)=-y_{k}+(-1)^{k+1} \nu \ell_{k}
$$

and

$$
\forall k \in \mathbb{N}^{*}: u_{k}(0)=(-1)^{k} \nu \sum_{i=0}^{k-1} \ell_{i} .
$$

Thus, $u$ is bounded if and only if either $\nu=0$, and thereby $u=0$, or $\nu \neq 0$ and $L\left(\Gamma_{0}\right)<\infty$. Thus, $\Gamma_{0}$ is Liouville w.r. to $(K C)$ if and only if $L\left(\Gamma_{0}\right)=\infty$.

Next, we show three useful lemmata. If $\Theta$ is a subgraph of the graph $\Gamma$ then by zero extension, $\Pi^{+}(\Theta)$ and $\Pi_{\infty}^{+}(\Theta)$ are isomorphic to subspaces of $\Pi^{+}(\Gamma)$ and $\Pi_{\infty}^{+}(\Gamma)$, respectively. In particular,

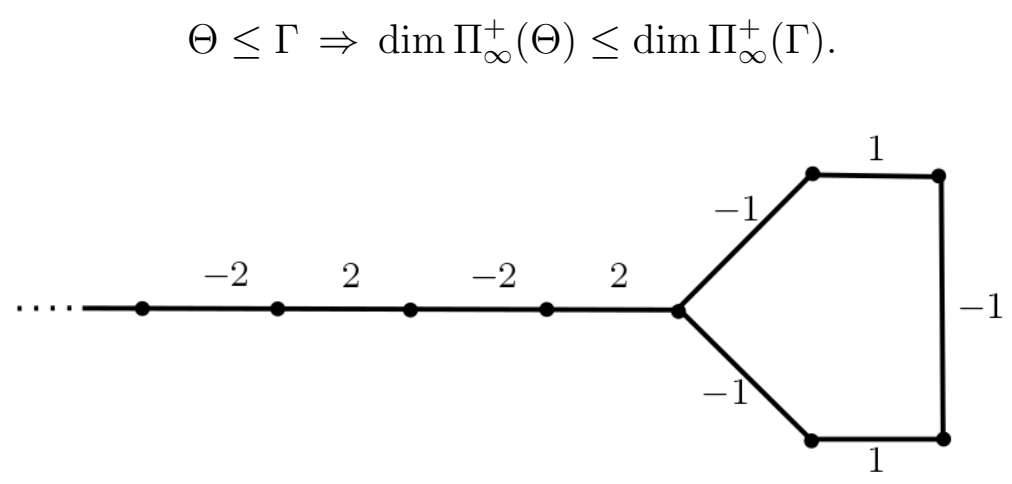

Figure 2: An infinite non bipartite graph with a nonvanishing element belonging to $\Pi_{\infty}^{+}(\Gamma)$. 
Infinite and even finite circuits $\zeta$ fulfill $\operatorname{dim} \Pi_{\infty}^{+}(\zeta)=1$. The same holds for a dumbbell $\delta$ composed by two odd finite circuits with at most one vertex in common or with a path joining them. Finally, a graph $\pi$ composed by an odd finite circuit $\zeta$ and an one-sided unbounded path $N \cong \Gamma_{0}$ with $V(\zeta) \cap V(N)=\left\{v_{0}\right\}$ and $\gamma\left(v_{0} ; N\right)=1$, satisfies $\operatorname{dim} \Pi_{\infty}^{+}(\pi)=1$, see Fig. 2 . Thus, we have shown the following

Lemma 3.2 A graph $\Gamma$ satisfies $\operatorname{dim} \Pi_{\infty}^{+}(\Gamma) \geq 1$ if it contains

(a) either an infinite circuit,

(b) or a finite even circuit,

(c) or two finite odd circuits with at most one vertex in common,

(d) or a graph $\pi$ composed by an odd finite circuit $\zeta$ and an one-sided unbounded path $N \cong \Gamma_{0}$ with $V(\zeta) \cap V(N)=\left\{v_{0}\right\}$ and $\gamma\left(v_{0} ; N\right)=1$.

In particular, $\Gamma$ cannot be a Liouville graph with respect to $(K C)$.

Lemma 3.3 Consider a vertex $v$ in some graph $\Gamma$, at which a finite tree $T$ is attached such that $V(\Gamma) \cap V(T)=\{v\}$ and such that $\gamma^{-}(v ; T)=0=\gamma^{+}(v ; T)-1$. Suppose that at all other vertices $w \in V(T)$ the outdegree satisfies $\gamma^{-}(w ; T)=1$. Let $\Sigma$ denote the composed graph by $\Gamma$ and $T$ and suppose $u \in \mathcal{H}_{K C}(\Sigma)$. Then on each edge $k_{j}$ of $T$, u satisfies

$$
\nu\left(\pi_{j}\left(\ell_{j}\right)\right) u_{j}\left(\ell_{j}\right) \geq 0
$$

Proof. For $\nu=0$, the assertion is plain. W.l.o.g. we can assume $\nu=1$. We always have

$$
u_{k}\left(x_{k}\right)=u_{k}(0)-\nu(\pi(0)) x_{k}=u_{k}(0)+\nu\left(\pi\left(\ell_{k}\right)\right) x_{k},
$$

in particular

$$
\nu\left(\pi\left(\ell_{k}\right)\right) u_{k}\left(\ell_{k}\right)=\nu\left(\pi_{k}\left(\ell_{k}\right)\right) u_{k}(0)+\ell_{k}=-\nu\left(\pi_{k}(0)\right) u_{k}(0)+\ell_{k} .
$$

If $\pi_{j}(0)$ is a boundary vertex, then evidently $\nu\left(\pi_{j}\left(\ell_{j}\right)\right) u_{j}\left(\ell_{j}\right)=\ell_{j}>0$. By recurrence on the distance to the boundary of $T$, we conclude

$$
\begin{aligned}
\nu\left(\pi_{j}\left(\ell_{j}\right)\right) u_{j}\left(\ell_{j}\right) & =\ell_{j}-\nu\left(\pi_{j}(0)\right) u_{j}(0) \\
& =\ell_{j}+\sum_{\pi_{j}(0)=\pi_{k}\left(\ell_{k}\right)} \nu\left(\pi_{k}\left(\ell_{k}\right)\right) u_{k}\left(\ell_{k}\right)>0 .
\end{aligned}
$$

Lemma 3.4 Let $\Gamma$ be a graph that contains as an induced subgraph $\Gamma_{0}$ such that at the vertices of the latter one finite induced trees in $\Gamma$ are eventually attached. Label the vertices of $\Gamma_{0}$ in $\Gamma$ by $\mathbb{N}$ as above by the natural incidences

$$
d_{i i}=-1, \quad d_{i+1, i}=1
$$


Suppose that

$$
\sum_{i=0}^{\infty} \ell_{i}=\infty
$$

Then $\Gamma$ has no bounded harmonic function with nonzero slopes.

Proof. Reasoning by contradiction, we suppose that there exists $u \in \mathcal{H}_{K C}^{\infty}(G)$ with $\nu \neq 0$. W.l.o.g. suppose $\nu=1$.

Let $T_{k}$ denote the eventual finite tree at $v_{k}$. Let $a_{k}$ denote the sum of the incoming values of $u$ at $v_{k}$ that do not belong to edges of $\Gamma_{0}$. As a harmonic function has to vanish on edges incident with boundary vertices, as $T_{k}$ does not contain circuits, and as by choosing the orientation "from the boundary of $T_{k}$ to $\Gamma_{0}$, the outdegree of each vertex in $V\left(T_{k}\right) \backslash V\left(\Gamma_{0}\right)$ amounts to 1 , each $a_{k}$ is uniquely determined. Moreover, by Lemma 3.3,

$$
\forall k \in \mathbb{N}: \nu_{k} a_{k} \geq 0 .
$$

On each edge of $\Gamma_{0}, u$ has the form

$$
u_{k}\left(x_{k}\right)=u_{k}(0)-\nu_{k} x_{k}=: y_{k}-\nu_{k} x_{k} .
$$

Then $(K C)$ leads to the recurrence

$$
y_{k+1}=-y_{k}-a_{k+1}+\nu_{k} \ell_{k} .
$$

Multiply (11) by $\nu_{k+1}$ and get with $z_{k}=\nu_{k} y_{k}$ the recurrence

$$
z_{k+1}=z_{k}-\nu_{k+1} a_{k+1}+\nu_{k} \nu_{k+1} \ell_{k}=z_{k}-\nu_{k+1} a_{k+1}-\ell_{k},
$$

in other words

$$
z_{k}=z_{0}-\sum_{i=1}^{k}\left(\nu_{i} a_{i}+\ell_{i-1}\right)
$$

Using (9) and (10), this shows that $\left|z_{k}\right|=\left|y_{k}\right| \rightarrow \infty$ as $|k| \rightarrow \infty$, in contradiction to the presumed bounded character of $u$. Thus, $\nu=0$.

Example 3.5 The two-sided unbounded path $\Gamma_{1}$ consists in the vertex set $\mathbb{Z}$ with edges defined by the natural incidences

$$
d_{i i}=-1, \quad d_{i, i-1}=1 .
$$

Clearly, $\operatorname{dim} \Pi_{\infty}^{+}\left(\Gamma_{1}\right)=1$. $\Gamma_{1}$ is Liouville w.r. to $(C K)$, but not w.r. to $(K C)$, since by Lemma 3.4 or more directly, using that $u \in \mathcal{H}_{K C}\left(\Gamma_{1}\right)$ is completely determined by its restriction to an arbitrary fixed edge,

$$
m\left(0 ; \Gamma_{1} ; K C\right)= \begin{cases}1 \quad \text { if } \sum_{k \in \mathbb{Z}} \ell_{k}=\infty & (\nu=0) \\ 2 \text { if } \sum_{k \in \mathbb{Z}} \ell_{k}<\infty & (\nu=0 \& \nu \neq 0)\end{cases}
$$




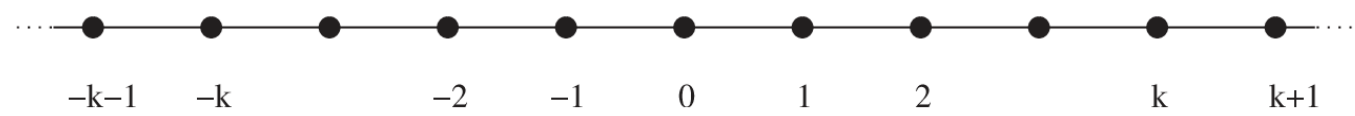

Figure 3: The two-sided unbounded path $\Gamma_{1}$.

\section{Infinite Liouville graphs and infinite trees without two-sided unbounded paths}

According to Lemma 2.1, the finite Liouville graphs under $(K C)$ are exactly trees and unicyclic non bipartite graphs. In the infinite case, we can state the following

Theorem 4.1 Let $\Gamma$ be a graph that contains a copy of $\Gamma_{0}$ whose edges satisfy

$$
\sum_{e \in E\left(\Gamma_{0}\right)} \ell(e)=\infty
$$

Then $\Gamma$ is a Liouville graph under $(K C)$ if and only if $\Gamma$ is a tree in the following list:

1. The one-sided unbounded path $\Gamma_{0}$,

2. a sole $\Gamma_{0}$ attached at its boundary vertex to a finite tree.

3. a sole $\Gamma_{0}$ with infinitely many finite trees attached to its vertices.

This applies in particular to the equal length case, or more generally, to the case $\inf \left\{\ell_{i} \mid i \in \mathbb{N}\right\}>0$.

Proof. The sufficiency is plain with Lemma 3.4. Next, suppose that $\Gamma$ is a Liouville graph. By Lemma 3.2, $\Gamma$ must be a tree and cannot contain copies of $\Gamma_{1}$. By hypothesis and $(12), \operatorname{dim} \Pi_{\infty}^{+}(\Gamma)=0$. Finally, the assertion follows with Lemma 3.4.

As already seen in Example 3.1, without Condition (12) the assertion is wrong, since $\Gamma_{0}$ admits bounded harmonic functions with $\nu \neq 0$ for $L\left(\Gamma_{0}\right)<\infty$. Does this also hold if to each vertex of $\Gamma_{0}$ finite trees are attached? Accordingly, suppose that $T$ is a tree containing $\Gamma_{0}$, but not $\Gamma_{1}$ and fulfilling

$$
L\left(\Gamma_{0}\right)=\sum_{e \in E\left(\Gamma_{0}\right)} \ell(e)<\infty
$$

Number the vertices of $\Gamma_{0}$ by $\mathbb{N}$ and choose the natural incidence

$$
d_{i i}=-1, \quad d_{i, i-1}=1 .
$$

on $\Gamma_{0}$. Clearly, $\Pi_{\infty}^{+}(T)=\{0\}$. We want to know, whether bounded harmonic functions with nonvanishing slope factor occur or not. Let $T_{k}$ denote the finite wood 
incident with $v_{k}$, but having no edges with $\Gamma_{0}$ in common. Let $u \in \mathcal{H}_{K C}(T)$ allow $\nu=\nu(u)>0$ and, as above, $a_{k}$ denote the sum of the incoming values of $u$ at $v_{k}$ on $T$ that do not belong to edges of $\Gamma_{0}$. Recall that each $a_{k}$ is uniquely determined for given $\nu$. W.l.o.g. choose $\nu=1$ and

$$
\nu_{k}=-(-1)^{k}
$$

set $y_{k}:=u_{k}(0)$ and get $u_{k}\left(x_{k}\right)=y_{k}+(-1)^{k} x_{k}$. Then $(K C)$ defines the linear recurrence

$$
y_{0}=-a_{0}, \quad \forall k \in \mathbb{N}^{*}: y_{k+1}=-y_{k}-(-1)^{k} \ell_{k}-a_{k+1} .
$$

The solution of (14) takes the form

$$
y_{k}=(-1)^{k}\left(\sum_{i=0}^{k-1} \ell_{i}-a_{0}+\sum_{i=1}^{k}(-1)^{i+1} a_{i}\right)=(-1)^{k}\left(\sum_{i=0}^{k} \nu_{i} a_{i}+\sum_{i=0}^{k-1} \ell_{i}\right),
$$

Lemma 3.3 shows that all $\nu_{k} a_{k}$ are nonnegative. Thus, under (13)

$$
u \in \mathcal{H}_{K C}^{\infty}(T) \Longleftrightarrow \sum_{k=0}^{\infty}\left|a_{k}\right|<\infty .
$$

This yields examples of trees $T$ fulfilling (13) that are not Liouville graphs. Take $\nu=1$ and $\Gamma_{0}$ with edge lengths just fulfilling (13). Or add to at each vertex $v_{i}$ of $\Gamma_{0}$ just one additional edge of length $\frac{1}{(k+1)^{2}}$ with its boundary vertex as initial node. Then

$$
\left|y_{k}\right| \leq \frac{\pi^{6}}{6}+\sum_{i=0}^{\infty} \ell_{i}<\infty
$$

Thus, $u$ defines a bounded harmonic function with nonvanishing slope factor.

But (16) yields also examples that even under (13), $T$ can be a Liouville graph. If each $T_{k}$ is just an edge of length 1 with $a_{k}=1$, see the graph on the 1.h.s in Fig. 4 , or if $T_{k}$ is a path of length $k+1$ with edge lengths 1 with

$$
\forall k \in \mathbb{N}: a_{k}=(-1)^{k}(k+1),
$$

see see the graph on the r.h.s in Fig. 4, then Formulae (15) and (16) show that $\nu=1$ is excluded for a bounded harmonic function. Thus, in both cases, $m(0 ; T ; K C)=0$.

Corollary 4.2 Suppose the tree $T$ consists in a copy $\Gamma_{0}$ whose edge lengths satisfy Condition (13) and the vertices $v_{k}$ of which finite woods $T_{k}$ are attached that have precisely the vertex $v_{k}$ with $\Gamma_{0}$ in common. If

$$
\sum_{i=0}^{\infty} L\left(T_{i}\right)<\infty
$$

then $\mathcal{H}_{K C}(T)$ contains bounded elements with arbitrary nonvanishing slope factor $\nu$. In particular, $T$ is not a Liouville graph. 


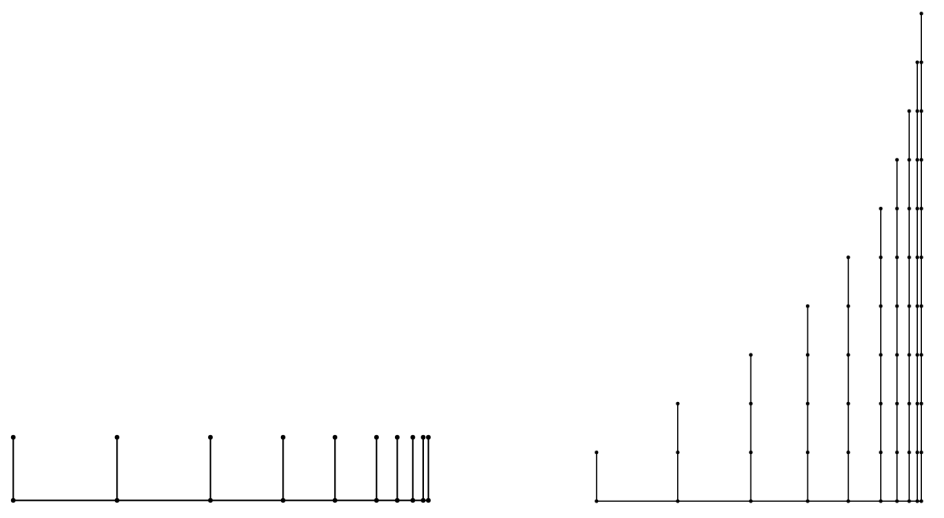

Figure 4: Two infinite Liouville trees with (13).

Proof. By recurrence on the number of edges of $T_{k}$ it follows that

$$
\left|a_{k}\right| \leq|\nu| L\left(T_{k}\right)
$$

Now (16) permits to conclude that there exists $u \in \mathcal{H}_{K C}^{\infty}(T)$ with $\nu(u)=1$, which, in turn, yields the assertion.

\section{Medusae}

By definition, an infinite graph $\Gamma$ is called a medusa if it has only finitely many essential ramification nodes,

$$
\# V_{\mathrm{ess}}(\Gamma)<\infty
$$

Under $(C K)$, a medusa is a Liouville graph, see [3]. For such a graph $\Gamma$ we shall adopt the following notation throughout this section. Let $B \leq \Gamma$ denote the smallest finite connected induced subgraph such that

$$
V_{\mathrm{ess}}(\Gamma) \subset V(B)
$$

Moreover, let $\Gamma_{0}^{1}, \ldots, \Gamma_{0}^{N} \leq \Gamma$ denote the $N$ disjoint subgraphs isomorphic to $\Gamma_{0}$. For each $\Gamma_{0}^{k}$ let $v_{0}^{k}$ denote its boundary vertex and $e_{0}^{k}$ its incident edge in $\Gamma_{0}^{k}$. We can choose these nodes to fulfill for each $1 \leq k \leq N$

$$
\left\{v_{0}^{k}\right\}=V(B) \cap V\left(\Gamma_{0}^{k}\right)
$$

Then we can state

Theorem 5.1 Suppose that $\Gamma$ is a medusa that either fulfills $L(G)=\infty$, or that is non bipartite with $L(G)<\infty$. Then all slope factors $\nu$ of bounded harmonic functions on $G$ vanish and

$$
\operatorname{dim} \Pi^{+}(B)+N-1 \leq m(0 ; \Gamma ; K C) \leq \operatorname{dim} \Pi^{+}(B)+N
$$


Proof. Necessarily $\nu=0$ by (7). The lower bound follows readily with Lemma 3.2, first applied to $\Pi^{+}(B)$ considered as a subspace of $\Pi_{\infty}^{+}(\Gamma)$. Secondly, each pair $\Gamma_{0}^{k}$ and $\Gamma_{0}^{j}$ forms an infinite circuit $\Gamma_{1}^{k, j}$ isomorphic to $\Gamma_{1}$. Among those exactly $N-1$, say $\Gamma_{1}^{2,1}, \ldots, \Gamma_{1}^{N, 1}$, are the supports of linearly independent elements belonging to $\Pi_{\infty}^{+}(\Gamma)$. As each $\varphi \in \Pi_{\infty}^{+}(\Gamma)$ with support $\Gamma_{1}^{k, j}$ can be written as a linear combination of two such elements with supports $\Gamma_{1}^{1, k}$ and $\Gamma_{1}^{1, j}$, the lower bound is shown.

As for the upper bound, suppose first that $N=1$. If there is $\varphi \in \Pi_{\infty}^{+}(\Gamma)$ such that $\varphi\left(v_{0}^{1}\right)=1$, then each harmonic function can be written as

$$
u=\underbrace{u-u\left(v_{0}^{1}\right) \varphi}_{\in \Pi^{+}(B)}+\underbrace{u\left(v_{0}^{1}\right) \varphi}_{\in\langle\varphi\rangle} .
$$

As the restriction of $u$ to $\Gamma_{0}^{1}$ is uniquely determined by $u\left(v_{0}^{1}\right), u-u\left(v_{0}^{1}\right) \varphi$ belongs to $\Pi^{+}(B)$. This shows $m(0 ; \Gamma ; K C) \leq \operatorname{dim} \Pi^{+}(B)+1$.

If there is no $\varphi \in \Pi_{\infty}^{+}(\Gamma)$ such that $\varphi\left(v_{0}^{1}\right)=1$, then all elements of $\Pi_{\infty}^{+}(\Gamma)$ have their supports in $B$ and $m(0 ; \Gamma ; K C)=\operatorname{dim} \Pi^{+}(B)$. Thus, the assertion is shown for $N=1$.

For $N \geq 2$, there exists always $\varphi \in \Pi_{\infty}^{+}(\Gamma)$ such that $\varphi\left(v_{0}^{N}\right)=1$. Let $\tilde{\Gamma}$ denote the graph by removing the edges of $\Gamma_{0}^{N}$ from $\Gamma$ while keeping $v_{0}^{N} \in V(\tilde{\Gamma})$. Again, as the restriction to $\Gamma_{0}^{N}$ of each bounded harmonic function $u$ is uniquely determined by $u\left(v_{0}^{N}\right), u$ reads

$$
u=\underbrace{u-u\left(v_{0}^{N}\right) \varphi}_{=: w}+\underbrace{u\left(v_{0}^{N}\right) \varphi}_{\in\langle\varphi\rangle}
$$

where $w$ vanishes on $\Gamma_{0}^{N}$ and can be considered as an element of $\mathcal{H}_{K C}^{\infty}(\tilde{G})$ by restriction to $\tilde{\Gamma}$. By recurrence on $N, m(0 ; \Gamma ; K C) \leq \operatorname{dim} \Pi^{+}(B)+N-1+1$.

The examples at the end of this section will show that both bounds in Theorem 5.1 are optimal. However, in the bipartite case the upper bound is always attained when the total graph length is finite.

Theorem 5.2 Suppose $\Gamma$ is a bipartite medusa with $L(G)<\infty$. Then there are bounded harmonic functions on $G$ with nonvanishing slope factor and

$$
m(0 ; \Gamma ; K C)=\operatorname{dim} \Pi^{+}(B)+N .
$$

Proof. (a) Let us show first that for each $0 \neq \nu \in \mathbb{R}$, there exists $u \in \mathcal{H}_{K C}^{\infty}(G)$ such that $\nu(u)=\nu$. Clearly, w.l.o.g. we can choose $\nu=1$. As $\Gamma$ is bipartite, we can endow it with the sink-source-orientation, i.e. each vertex $v$ of $\Gamma$ either fulfills $\gamma(v)=\gamma^{-}(v)$ or $\gamma(v)=\gamma^{+}(v)$. Thus, a presumed bounded harmonic function $u$ is on each edge of $B$ the form

$$
u_{j}\left(x_{j}\right)=c_{j}+x_{j}
$$

with some $c_{j} \in \mathbb{R}$ for $1 \leq j \leq m:=\# E(B)$. The $m$-column vector $c=\left(c_{j}\right)_{m \times 1}$ satisfies the following $n:=\# V(B)$ equations. Let $\alpha_{0}^{k}$ denote the presumed value of $u$ on $e_{0}^{k}$ in $\Gamma_{0}^{k}$. At each vertex $v_{i}$ set 


$$
s_{i}=\sum_{v_{i} \in k_{j}} \ell_{j} .
$$

Then at each source $v_{i} \in V(B)$ with $\nu_{i}=-1$,

$$
\sum_{v_{i}=\pi_{j}(0)} c_{j}= \begin{cases}0 & \text { if } v_{i} \notin\left\{v_{0}^{k} \mid 1 \leq k \leq N\right\}, \\ -\alpha_{0}^{k} & \text { if } v_{i}=v_{0}^{k},\end{cases}
$$

and at each sink $v_{i} \in V(B)$ with $\nu_{i}=1$,

$$
\sum_{v_{i}=\pi_{j}\left(\ell_{j}\right)} c_{j}= \begin{cases}-s_{i} & \text { if } v_{i} \notin\left\{v_{0}^{k} \mid 1 \leq k \leq N\right\}, \\ -\alpha_{0}^{k}-s_{i} & \text { if } v_{i}=v_{0}^{k} .\end{cases}
$$

In other words, if $\mathcal{D}^{+}$denotes the unsigned $n \times m$-incidence matrix of $B$, then

$$
\mathcal{D}^{+} c=b:=-s^{+}-\sum_{v_{i}=v_{0}^{k}} \alpha_{0}^{k} \mathbf{e}_{i}^{n}
$$

with

$$
s^{+}=\left(s_{i}^{+}\right)_{n \times 1}, \quad s_{i}^{+}=\left\{\begin{array}{ll}
s_{i} & \text { if } v_{i} \text { is a sink, } \\
0 & \text { otherwise }
\end{array},\right.
$$

admitting the $N \leq n$ parameters $\alpha_{0}^{k}$. But $\operatorname{rank}\left(\mathcal{D}^{+}\right)=n-1$. Thus, it remains to show that the parameters $\alpha_{0}^{k}$ can be chosen such that the image of $\mathcal{D}^{+}$and the affine subspace $-s^{+}-\bigoplus_{v_{i}=v_{0}^{k}} \mathbb{R} \mathbf{e}_{i}^{n}$ are not parallel.

But, it suffices to show the case $N=1$, since eventual additional $\Gamma_{0}^{k}, k \geq 2$, allow $\alpha_{0}^{k}=0$ and $\partial_{0} u_{0}^{k}(0)= \pm \nu$ together with the shown case $N=1$. However, $\alpha_{0}^{1}=0$ is excluded, since $B$ alone does not allow bounded harmonic functions with $\nu \neq 0$ by Section 2. W.l.o.g. we can assume that $v_{1}=v_{0}^{1}$ and $\nu_{1}=-1$. Then the r.h.s. in (18) reduces to $b=-\alpha_{0}^{1} \mathbf{e}_{1}^{n}-s^{+}$. As $\operatorname{rank} \mathcal{D}^{+}(B)=n-1$, we find $h \in \mathbb{R}^{n}$ such that

$$
\mathcal{D}^{+}\left(\mathbb{R}^{m}\right)=\langle h\rangle^{\perp} \quad \text { and } \quad(h, h)_{2}=1,
$$

where $(\cdot, \cdot)_{2}$ denotes the Euclidean scalar product in $\mathbb{R}^{n}$. Lemma 8.2 guarantees that $\left(s^{+}, h\right)_{2} \neq 0$, since $s^{+}$cannot belong to $\operatorname{Im} \mathcal{D}^{+}(B)$. If $\left(h, \mathbf{e}_{1}^{n}\right)_{2} \neq 0$, then for

$$
\alpha_{0}^{1}=-\frac{\left(s^{+}, h\right)_{2}}{\left(h, \mathbf{e}_{1}^{n}\right)_{2}}
$$

$b \in \mathcal{D}^{+}\left(\mathbb{R}^{m}\right)$, and its preimage defines the desired vector $c=\left(c_{j}\right)_{m \times 1}$.

Finally, if $\left(h, \mathbf{e}_{1}^{n}\right)_{2}=0$, then $\mathbf{e}_{1}^{n} \in \mathcal{D}^{+}\left(\mathbb{R}^{m}\right)$, which is excluded by (8.2). Thus, the existence of $u \in \mathcal{H}_{K C}^{\infty}(G)$ with $\nu(u)=1$ is well established. 
Next we show the formula for $m(0 ; \Gamma ; K C)$ and suppose first that

$$
N=1 \text {. }
$$

(b) There is no $u \in \mathcal{H}_{K C}^{\infty}(\Gamma)$ such that $\nu(u) \neq 0$ and $u\left(v_{0}^{1}\right)=0$. Otherwise, by (6), the restriction of $u$ to $B$ would constitute an element of $\mathcal{H}_{K C}(B)$ with nonzero slope, which is excluded in the finite case as above, see Section 2.

(c) By (a) there exists $u \in \mathcal{H}_{K C}^{\infty}(\Gamma)$ with nonzero slope factor $\nu(u)$. Thus, there is no $\varphi \in \Pi_{\infty}^{+}(\Gamma)$ such that $\varphi\left(v_{0}^{1}\right)=1$. Otherwise, each $u$ as above could be written in the form

$$
u=\underbrace{u-u\left(v_{0}^{1}\right) \varphi}_{=: w}+\underbrace{u\left(v_{0}^{1}\right) \varphi}_{\in \Pi_{\infty}^{+}(\Gamma)}
$$

with $w \in \mathcal{H}_{K C}^{\infty}(\Gamma)$ and $\nu(w)=\nu(u) \neq 0$ and $v\left(w_{0}^{1}\right)=0$, in contradiction to (b). Conclusion: All elements of $\Pi_{\infty}^{+}(\Gamma)$ have their supports in $B$ and

$$
\Pi_{\infty}^{+}(\Gamma) \cong \Pi^{+}(B) .
$$

(d) Each element $u \in \mathcal{H}_{K C}^{\infty}(\Gamma)$ with $\nu(u) \neq 0$ is uniquely determined by $u\left(v_{0}^{1}\right)$. Thus, if $\tilde{u} \in \mathcal{H}_{K C}^{\infty}(\Gamma)$ with $\nu(\tilde{u})=\nu(u)$ and $u\left(v_{0}^{1}\right)=\tilde{u}\left(v_{0}^{1}\right)$, then $u-\tilde{u}$ vanishes on $\Gamma_{0}$. If $u$ and $\tilde{u}$ were different, then $u-\tilde{u}$ would constitute a bounded harmonic function on $B$ with slope factor $\nu(u) \neq 0$, which is excluded by Section 2. Thus,

$$
\operatorname{dim}\left\langle u \in \mathcal{H}_{K C}^{\infty}(\Gamma) \mid \nu(u) \neq 0\right\rangle=1,
$$

and, thereby with $(\mathrm{c}), m(0 ; \Gamma ; K C)=\operatorname{dim} \Pi^{+}(B)+1$.

(e) For

$$
N \geq 2 \text {, }
$$

again there exists always $\varphi \in \Pi_{\infty}^{+}(\Gamma)$ such that $\varphi\left(v_{0}^{N}\right)=1$. Thus, each $u \in \mathcal{H}_{K C}^{\infty}(G)$ can be written as

$$
u=\underbrace{u-u\left(v_{0}^{N}\right) \varphi}_{=: w}+\underbrace{u\left(v_{0}^{N}\right) \varphi}_{\in\langle\varphi\rangle} .
$$

As above, let $\tilde{\Gamma}$ denote the graph by removing the edges of $\Gamma_{0}^{N}$ from $\Gamma$ while keeping $v_{0}^{N} \in V(\tilde{\Gamma})$. The slope factor of the restriction of $w$ to $\tilde{\Gamma}$ determines uniquely the one of $u$ on the whole medusa and, thereby, $\left\{w \in \mathcal{H}_{K C}^{\infty}(G) \mid w\left(v_{0}^{N}\right)=0\right\}$ is isomorphic to a subspace of $\mathcal{H}_{K C}^{\infty}(\tilde{G})$. By recurrence,

$$
m(0 ; \Gamma ; K C)=\operatorname{dim} \Pi^{+}(B)+N-1+1 .
$$

The proofs of Theorems 5.1 and 5.2 illustrate very well that the case $N=1$ is more delicate than higher numbers $N$ of "tentacles" in the medusa. We illustrate this also with the aid of some examples.

The minimal medusa is just $\Gamma_{0}$ with $\Pi_{\infty}^{+}\left(\Gamma_{0}\right)=\{0\}$. If $L\left(\Gamma_{0}\right)<\infty$, then $m\left(0 ; \Gamma_{0} ; K C\right)=1$, and the only nontrivial bounded harmonic functions are the ones with $u_{0}(0)=0$ and $\nu(u) \neq 0$. 
Example 5.3 If $B$ is tree and $L(G)=\infty$, then $\operatorname{dim} \Pi^{+}(B)=0$ and $\Gamma$ is a Liouville graph for $N=1$ by Theorem 4.1. For $N \geq 1, m(0 ; \Gamma ; K C)=N-1$. See e.g. the infinite star graph in Fig. 9. This shows that, in general, the upper bound $\operatorname{dim} \Pi^{+}(B)+N$ in Theorem 5.1 is not attained. But for $L(G)<\infty$, the upper bound is well attained since $m(0 ; \Gamma ; K C)=N$ by Theorem 5.2. Here we find exactly one additional independent bounded harmonic function with $\nu \neq 0, \operatorname{since} \operatorname{corank}(\Gamma)=0$.

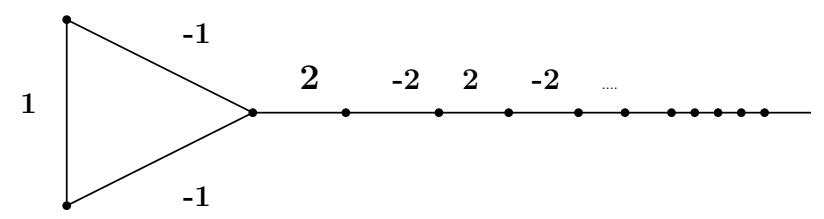

Figure 5: A non bipartite medusa displaying a nonvanishing bounded harmonic function stemming from the generalized unsigned circuit space.

Example 5.4 Compose a medusa $\Gamma$ by joining an odd circuit $C$ and one copy of $\Gamma_{0}$, see Fig. 5. Clearly $\nu=0$, and independently of $L\left(\Gamma_{0}\right), \Pi^{+}(C) \cong\{0\} \cong \Pi_{\infty}^{+}\left(\Gamma_{0}\right)$, but $m(0 ; \Gamma ; K C)=1$, since $\operatorname{dim} \Pi_{\infty}^{+}(\Gamma)=1$. This example also displays that, in general, the vector space $\Pi_{\infty}^{+}$cannot be generated by the ones of covering induced subgraphs. Mutatis mutandis, for any non bipartite unicyclic graph $B$, we have $\Pi^{+}(B) \cong\{0\} \cong \Pi_{\infty}^{+}\left(\Gamma_{0}\right)$, but

$$
m(0 ; \Gamma ; K C)=N .
$$

A basis of $\mathcal{H}_{K C}^{\infty}(G)$ is readily obtained by choosing for each $\Gamma_{0}^{k}$ an element as in Fig. 5 and by extending it trivially to the remaining edges of $\Gamma$.

Example 5.5 Compose a medusa $\Gamma$ by joining an even circuit $C$ and one copy of $\Gamma_{0}$. For $L\left(\Gamma_{0}\right)=\infty, m(0 ; \Gamma ; K C)=1$, while for $L\left(\Gamma_{0}\right)<\infty, m(0 ; \Gamma ; K C)=2$, since on the one side $\operatorname{dim} \Pi_{\infty}^{+}(C)=1$ and each $\varphi \in \Pi_{\infty}^{+}(\Gamma)$ has its support in $C$, while on the other there is one independent $u \in \mathcal{H}_{K C}^{\infty}(G)$ with $\nu(u)=1$, that is well displayed in Fig. 6. Note that $\bullet$ stands for a vertex with $\nu_{i}>0$, while $\circ$ stands for $\nu_{i}<0$.

Example 5.6 Compose a medusa $\Gamma$ by attaching two copies of $\Gamma_{0}$ with $L\left(\Gamma_{0}\right)<\infty$ to a circuit $C$ with 4 vertices as depicted above. Then $m(0 ; \Gamma ; K C)=4$, since on the one side $\operatorname{dim} \Pi_{\infty}^{+}(\Gamma)=2$, while on the other there are two independent $u \in \mathcal{H}_{K C}^{\infty}(G)$ with $\nu(u)=1$, that are displayed in the Fig. 7, where again $\bullet$ stands for a vertex with $\nu_{i}>0$, while $\circ$ stands for $\nu_{i}<0$. Note that two incident values at one of the nodes of degree 3 determine $u$ completely. 


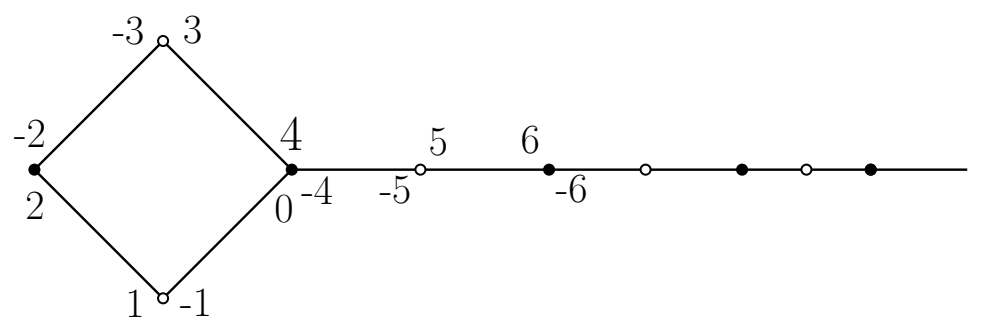

Figure 6: A bipartite medusa allowing the slope factor 1.
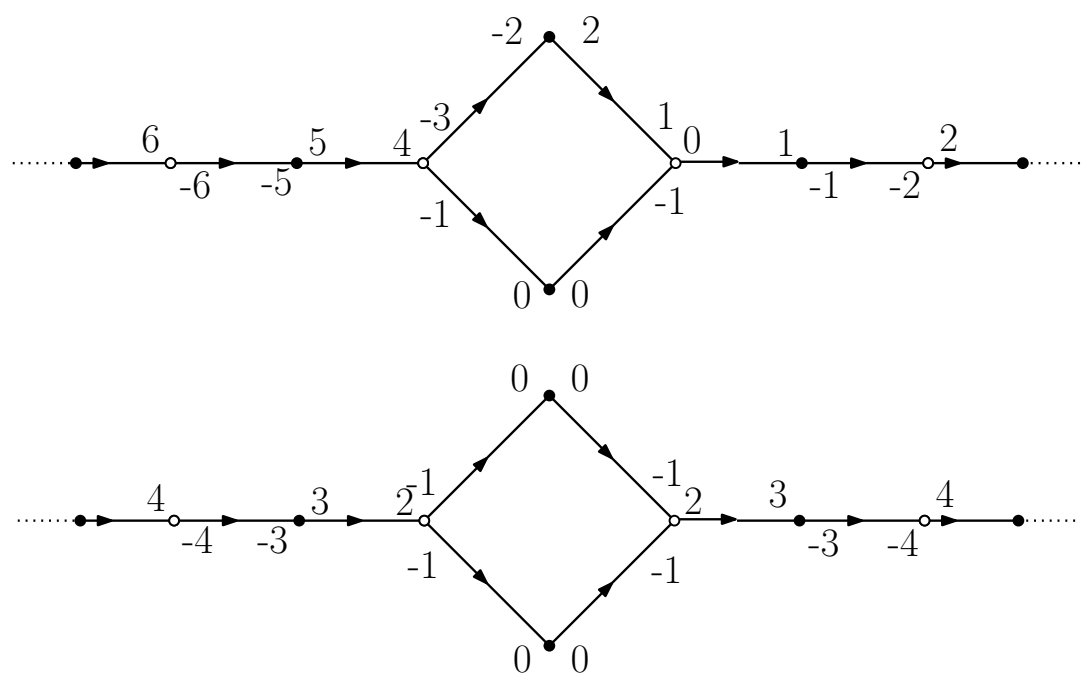

Figure 7: Two independent bounded harmonic functions with slope factor 1. 


\section{Trees}

Under $(C K)$, the condition

$$
\inf \left\{\ell_{j} \mid j \in \mathbb{N}\right\}>0 \text {. }
$$

plays a key role for the multiplicity of independent bounded harmonic functions. Moreover, the equivalence in Theorem 6.2 requires a supplementary condition on the edge length ratios. Under $(K C)$, no restriction is needed, only the notion of the reduced graph. Let us recall its construction. By definition, a viaduct in a graph $\Gamma$ is a path $\pi$ of length at least 2 in $\Gamma$ joining two distinct vertices $u$ and $v$ such that all other vertices in $V(\pi) \backslash\{u, v\}$ have the valency 2 in $\Gamma$ too.

Definition 6.1 The reduced graph $\Gamma_{\text {red }}$ of a given graph $\Gamma$ is constructed as follows. Introduce the operations

(I) Withdraw all edges in $\Gamma$ incident to boundary vertices.

(II) Withdraw each one-sided unbounded path $\pi$ in $\Gamma$ whose ramification nodes $V_{r}(\pi)$ are all nodes of valency 2 in $\Gamma$.

(III) Replace any viaduct $\pi$ in $\Gamma$ by a single edge of length $l$, where $l$ is the sum of the lengths of all edges of $\pi$.

Repeat (I) and (II) until there are no more vertices of valency 1 and no more onesided unbounded paths as in (II) in the remaining graph. Then apply (III) such that there are no more vertices of valency 2. The resulting graph is called the reduced graph $\Gamma_{\text {red }}$ of $\Gamma$.

Note that $\Gamma_{\text {red }}$ can reduce to a single vertex without edges, e.g. for $\Gamma=\Gamma_{1}$, or for any tree containing at most one copy of $\Gamma_{1}$ up to translation. As for the $(C K)$-condition, denote the minimal valency of a graph $\Gamma$ by $\gamma_{\min }(\Gamma)$ and introduce

$$
L(\Gamma)=\inf \left\{\frac{\text { arc length of } e}{\text { arc length of } f} \mid e, f \in E(\Gamma), e \cap f \neq \emptyset\right\} .
$$

Then we can cite the following

Theorem 6.2 [3, Theorem 5.5] Suppose that $T$ is a tree satisfying (19) such that

$$
L\left(T_{\text {red }}\right)>\frac{1}{\gamma_{\min }\left(T_{\text {red }}\right)-1} .
$$

Then

$$
m(0 ; T ; C K)=\infty \Longleftrightarrow \# V\left(T_{\text {red }}\right)=\infty
$$

In particular, the tree $T$ is a Liouville under $(C K)$ tree if and only if $\# V\left(T_{\text {red }}\right)<\infty$. 
Condition (20) is optimal. Already for 3-regular trees with $L(T)=\frac{1}{2}$ the assertion can be wrong, see [3, Example 5.9]. Note that for $\gamma_{\min } \geq 3$, Condition (20) reduces $L(T)>\frac{1}{\gamma_{\min }(T)-1}$.

As for the anti-Kirchhoff law, the situation is quite different. First note the following fact.

Lemma 6.3 A tree $T$ satisfies $\# V_{\mathrm{ess}}\left(T_{\mathrm{red}}\right)=\infty$ if and only if its reduced tree contains a copy of the 3-regular tree $T_{3}$.

Theorem 6.4 Let $T$ be a tree. Then $m(0 ; T ; K C)=\infty$ if and only if

$$
\# V_{\text {ess }}\left(T_{\text {red }}\right)=\infty \text {. }
$$

Proof. If $\# V_{\text {ess }}\left(T_{\text {red }}\right)=\infty$, then $T$ contains infinitely many finally independent copies of $\Gamma_{1}$. Each of them gives rise to a nonvanishing element of $\Pi_{\infty}^{+}(T)$. Conversely, $\# V_{\text {ess }}\left(T_{\text {red }}\right)<\infty$ implies that $T$ contains only finitely many copies of $\Gamma_{1}$ that are almost disjoint. As the supports of nonvanishing harmonic functions contain at least $\Gamma_{1}, m(0 ; T ; K C)<\infty$

But, bounded harmonics with nonzero slope $\nu$ can occur in trees containing the 3-regular tree $T_{3}$.

Example 6.5 Choose $\nu=1$. Consider the 3-regular tree $T_{3}$ with equal edge lengths 1. Endow $T_{3}$ with the $1 \mathrm{in}, 2$ out orientation. Choose any edge $k_{0}$ in $T_{3}$ and number the edges in the $\log _{2}(\cdot)$ backwards genealogical way. First, consider the vertex $\pi_{0}(0)$ as a root of the tree $T_{0}$ containing $k_{0}$ and all edges of $T_{3}$ being connected to $\pi_{0}(1)$ without passing by $\pi_{0}(0)$. Define a harmonic function $u \in \mathcal{H}_{K C}(T)$ as follows. Set

$$
u_{0}(0)=0, \quad u_{0}(1)=1, \quad u_{1}(0)=-\frac{1}{2}=u_{2}(0) .
$$

Recursively in the $K$-th generation with $K \geq 1$, with an edge $k_{j}$ with slope 1 ,

$$
2^{K-1}+1 \leq j+2 \leq 2^{K}
$$

and

$$
u_{j}(0)=\frac{2^{2 K}-1}{2^{2 K}},
$$

on the edges $k_{j+1}$ and $k_{j+2}$ with

$$
\pi_{j}(1)=\pi_{j+1}(0)=\pi_{j+2}(0)
$$

set

$$
\begin{aligned}
& \text { (a) } u_{j}(1)=\frac{2^{2 K+1}-1}{2^{2 K}}, \\
& \text { (b) } u_{j+1}(0)=u_{j+2}(0)=-\frac{2^{2 K+1}-1}{2^{2 K+1}}, \\
& \text { (c) } u_{j+1}(1)=u_{j+2}(1)=-\frac{2^{2(K+1)}-1}{2^{2 K+1}}, \\
& \text { (d) } u_{j+3}(0)=\ldots=u_{j+6}(0)=\frac{2^{2(K+1)}-1}{2^{2(K+1)} .}
\end{aligned}
$$




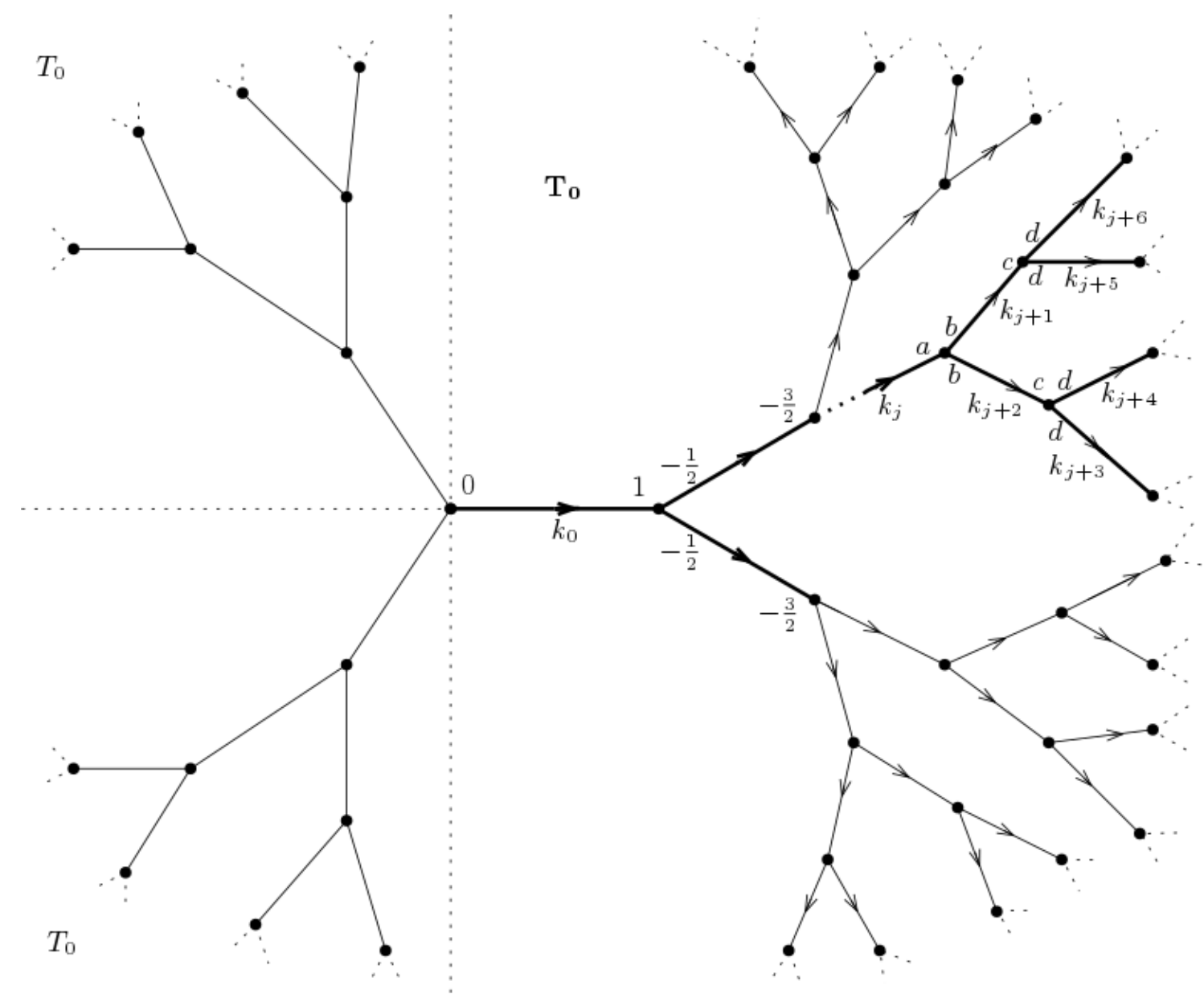

Figure 8: The 3-regular tree displaying a harmonic bounded function with $\nu=1$.

The letters $a, b, c, d$ stand for the corresponding values on the edges as indicated in Fig. 8. On the two copies of $T_{0}$ at $\pi_{0}(0)$ repeat the same construction. Then

$$
u \in L^{\infty}(T) \cap \mathcal{H}(T) .
$$

Clearly, at all the nodes, $\nu_{i}= \pm 1$.

Under $(C K)$, without (19), any finite multiplicity $m(0 ; T ; C K)$ occurs. But, under (19), there is only the following alternative.

Theorem 6.6 [5] A tree T satisfying (19) either is a Liouville graph under $(C K)$ or satisfies $m(0 ; T ; C K)=\infty$.

Under $(K C)$ this is no longer true, even under (19). Take the infinite star $\Sigma$ with equal edge lengths, $V_{\text {ess }}(\Sigma) \subset\left\{v_{0}\right\}$ and $N \geq 1$ copies of $\Gamma_{0}$ intersecting precisely in $v_{0}$. Then

$$
\operatorname{dim} \Pi_{\infty}^{+}(\Sigma)=N-1=m(0 ; \Sigma ; K C) .
$$




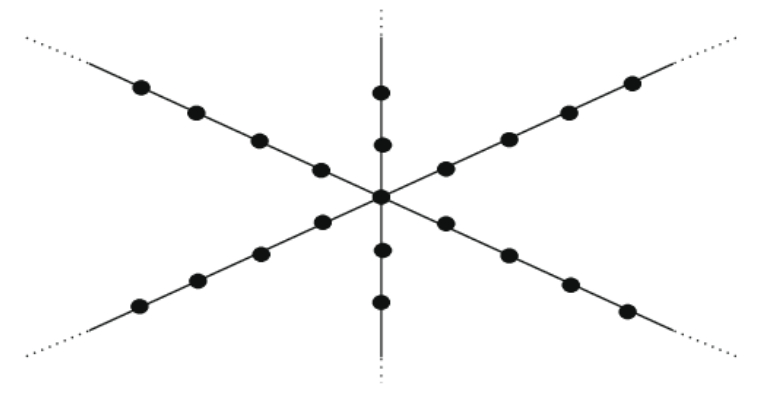

Figure 9: The infinite star with equal edge lengths.

\section{$7 \quad$ Graphs fulfilling $\mathbf{m}(\mathbf{0} ; \Gamma ; \mathrm{KC})=1$ and periodic graphs}

First, we characterize graphs with one independent bounded harmonic function satisfying $(K C)$.

Theorem 7.1 Let $\Gamma$ be a graph that contains a copy of $\Gamma_{0}$ with $L\left(\Gamma_{0}\right)=\infty$. Then $m(0 ; \Gamma ; K C)=1$ if and only if $\Gamma$ is a graph of the following list:

1. a sole induced one-sided unbounded path $\Gamma_{0}$ attached at its boundary vertex to a finite unicyclic graph,

2. a graph of Type 1 with finite trees attached to the vertices of $\Gamma_{0}$,

3. the two-sided unbounded path $\Gamma_{1}$ or $\Gamma_{1}$ with finite trees attached to its vertices.

In all these cases, there is no bounded harmonic function with nonzero slope factor.

Proof. Suppose $m(0 ; \Gamma ; K C)=1$. By hypothesis, $\Gamma$ can neither contain two finally disjoint copies of $\Gamma_{1}$, nor $\Gamma_{1}$ and a finite circuit, nor two finite circuits. Thus, if $\Gamma$ contains $\Gamma_{1}$, then it is a tree and contains exactly one copy of $\Gamma_{1}$ up to translation, at the vertices $v_{k}$ of which there might be finite trees $T_{k}$, which leads to the third case.

If $\Gamma$ contains an odd circuit $\eta$, then there cannot be a bounded harmonic function with nonzero slope by (7), and there is exactly one independent bounded harmonic function belonging to $\Pi_{\infty}^{+}(\Gamma)$ as defined in Fig. ?? and extended by 0 outside the circuit $\eta$ and outside $\Gamma_{0}$.

If $\Gamma$ contains an even circuit $\zeta$, then $\Pi_{\infty}^{+}(\Gamma) \cong \Pi_{\infty}^{+}(\zeta)$, and there cannot be a bounded harmonic function with nonzero slope. All bounded harmonic functions belonging to $\Pi_{\infty}^{+}(\Gamma)$ have their supports in $\zeta$.

In both of the latter two cases, $\Gamma_{0}$ must be induced and can allow finite trees at its vertices. This leads to the first and the second case, since Lemma 3.4 excludes nonzero slopes of bounded harmonic functions and since each such a function cannot vanish on the sole circuit. 
Conversely, if $\Gamma$ is of Type 1 or 2 , then Lemma 3.4 permits to conclude that $\Pi_{\infty}^{+}(\Gamma) \cong \mathcal{H}_{K C}^{\infty}(G)$. Using the same constructions as above it then follows that $m(0 ; \Gamma ; K C)=1$.

If $\Gamma$ is of Type 3 , then Lemma 3.4 again permits to conclude that $\Pi_{\infty}^{+}(\Gamma) \cong$ $\mathcal{H}_{K C}^{\infty}(G)$. As a harmonic function has to vanish on the eventual finite trees attached to the vertices of $\Gamma_{1}$, it follows that $m(0 ; \Gamma ; K C)=1$.

Theorem 7.1 applies in particular to periodic trees. Let us recall the following definition.

Definition 7.2 A graph $\Gamma$ is called a generalized lattice or periodic of rank $m \geq 1$ with translation group

$$
G=\bigoplus_{i=1}^{m} \mathbb{Z} b_{i} \leq \operatorname{Aut}(\Gamma),
$$

with kernel $N$ and with cell $F$, if the following conditions hold:

(a) $\Gamma$ is connected.

(b) $N$ and $F$ are finite connected subgraphs of $\Gamma$.

(c) $V(N)^{G}=V(\Gamma)$.

(d) $\left\{b_{i} \mid 1 \leq i \leq m\right\}$ is a basis of the free abelian group $G$.

(e) $F=\overline{N \cup \bigcup_{i=1}^{m} N^{b_{i}}}$ and $E(F)^{G}=E(\Gamma)$.

(f) $\forall g, h \in G: \quad g \neq h \quad \Longrightarrow \quad V\left(N^{g}\right) \cap V\left(N^{h}\right)=\emptyset$.

For more details see $[2,8,12]$ and $[3,4]$ for the spectral aspects with respect to $\Delta^{C K}$. Clearly, periodic graphs cannot be a Liouville graph under $(K C)$ by Lemma 3.2 , since they contain copies of $\Gamma_{1}$. Note that it is conceivable to assume $m \geq 1$ here, since $m=0$ corresponds to the case of a finite graph. Furthermore, recall that an eigenvalue of an operator is called a black hole eigenvalue if its eigenspace contains a subspace isomorphic to the inseparable Banach space $\ell^{\infty}(\mathbb{N})$.

Theorem 7.3 Let $\Gamma$ be a periodic graph.

1. If $\operatorname{rank} \Gamma \geq 2$, then $m(0 ; \Gamma ; K C)=\infty$ and 0 is a black hole eigenvalue under $(K C)$.

2. The same holds in the case rank $\Gamma=1$, if $\Gamma$ contains circuits.

3. Finally, $m(0 ; \Gamma ; K C)<\infty$ if and only if $\Gamma$ is a periodic tree, i.e. a band without circuits. In that case, $m(0 ; \Gamma ; K C)=1$, and no nonzero slope factor is possible.

Proof. If a periodic graph contains an even circuit, then 0 is a black hole eigenvalue since it possesses eigenfunctions of compact support. If the periodic graph contains odd circuits, then either the dumbbell construction, or a kernel enlargement leading to even circuits [2] lead to the same conclusion. If a periodic graph does not contain circuits, then it contains exactly one copy of $\Gamma_{1}$, up to translation. And at each vertex of $\Gamma_{1}$ an eventual finite tree is attached in a certain periodic way. Moreover, by periodicity, Condition (12) is fulfilled, and Theorem 7.1 permits to conclude. 
Example 7.4 Let $T$ be the infinite comb with all $\ell_{j}=1$. Thus, $T$ consists in the path $\Gamma_{1}$ to the vertices of which a sole edge is attached. Denote the vertices and edges of the path $\Gamma_{1}$ by $\mathbb{Z}$ and by the incidence $d_{i i}=-1$ and $d_{i, i-1}=1$. Let us illustrate that there is no bounded harmonic function $u$ on $T$ with $\nu=1$ and that $m(0 ; T ; K C)=1$. Choose

$$
\nu_{i}=-(-1)^{i}
$$

Then

$$
\forall i \in \mathbb{Z}: u_{2(i+1)}(0)=u_{2 i}(0)+4 \quad \& \quad u_{2 i+1}(0)=u_{2 i-1}(0)-4,
$$

which clearly shows that $u$ has to be unbounded.

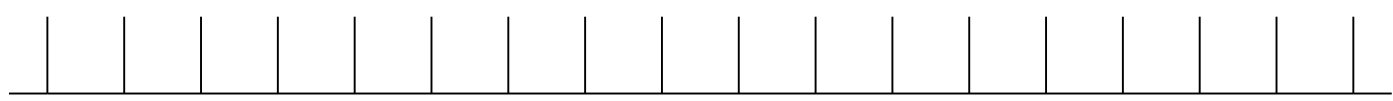

Figure 10: The infinite comb.

Example 7.5 Add to the foregoing example just one edge in a suitable kernel and get the periodic band $B$ generated by squares or just by one vertical edge. Again, we suppose that all $\ell_{j}=1$. Then $m(0 ; B ; K C)=\infty$ and 0 is in fact a black hole eigenvalue of $\Delta^{K C}$ with

$$
\mathcal{H}_{K C}^{\infty}(B) \cong \Pi_{\infty}^{+}(B) \cong \ell^{\infty}(\mathbb{Z})
$$

\begin{tabular}{|c|c|c|c|c|c|c|c|c|c|c|c|}
\hline$-x_{-1}$ & 0 & $-x_{0}$ & 0 & $-x_{1}$ & 0 & $-x_{2}$ & $\underline{0}$ & $-x_{3}$ & 0 & $-x_{4}$ & \\
\hline & $x_{-1} x_{0}$ & & $\begin{array}{ll}x_{0} & \\
& x_{1} \\
\end{array}$ & & $\begin{array}{ll}x_{1} & \\
& x_{2}\end{array}$ & & 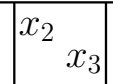 & & 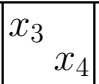 & & $x_{4}$ \\
\hline
\end{tabular}

This readily follows by associating to each sequence $\left(x_{k}\right)_{k \in \mathbb{Z}} \in \ell^{\infty}(\mathbb{Z})$ a unique element in $\Pi_{\infty}^{+}(B)$ as indicated in Fig. 11.

Figure 11: The periodic band $B$ with $\mathcal{H}_{K C}^{\infty}(B) \cong \ell^{\infty}(\mathbb{Z})$.

\section{Remarks and supplements}

\subsection{Length adjacency and anti-Kirchhoff law}

For the sake of simplicity we restrict ourselves to simple graphs, i.e. we exclude loops and multiple edges. In the $(C K)$ case, harmonic functions are closely related to a normalized adjacency operator. To be more specific, introduce

$$
\begin{gathered}
\mathcal{L}(\Gamma)=\left(\ell_{i h}\right)_{i, h \in \mathcal{I}}: \mathbb{R}^{V(\Gamma)} \longrightarrow \mathbb{R}^{V(\Gamma)}, \quad \mathcal{P}(\Gamma)=\left(\ell_{i h}^{-1}\right)_{i, h \in \mathcal{I}}: \mathbb{R}^{V(\Gamma)} \longrightarrow \mathbb{R}^{V(\Gamma)} \\
\ell_{i h}=\left\{\begin{array}{ll}
\ell_{s(i, h)} & \text { if } v_{i} \sim v_{h}, \\
0 & \text { otherwise },
\end{array} \quad s(i, h)= \begin{cases}s & \text { if } e_{s} \cap V=\left\{v_{i}, v_{h}\right\}, \\
1 & \text { otherwise. }\end{cases} \right.
\end{gathered}
$$


Then a continuous function $u$ on $G$ with $\mathbf{x}=\left(x_{i}\right)_{i \in \mathcal{I}}, x_{i}=u\left(v_{i}\right)$ belongs to $\mathcal{H}_{C K}(G)$ if and only if

$$
\operatorname{Diag}_{i}(\mathcal{P}(\Gamma) \mathbf{e})^{-1} \mathcal{P}(\Gamma) \mathbf{x}=\mathbf{x}
$$

In particular for equal edge lengths, (22) reduces to the classical mean value property

$$
\forall i \in \mathcal{I}: x_{i}=\frac{1}{\gamma_{i}} \sum_{v_{h} \sim v_{i}} x_{h}
$$

For the anti-Kirchhoff law $u \in \mathcal{H}_{K C}(G)$

$$
\begin{aligned}
0 & =\sum_{v_{i} \in k_{j}} u_{j}\left(v_{i}\right)=\sum_{v_{i}=\pi_{j}(0)} u_{j}(0)+\sum_{v_{i}=\pi_{j}\left(\ell_{j}\right)} u_{j}\left(\ell_{j}\right) \\
& =\sum_{v_{i}=\pi_{j}(0)}\left(u_{j}\left(\ell_{j}\right)-\ell_{j} \alpha_{j}\right)+\sum_{v_{i}=\pi_{j}\left(\ell_{j}\right)}\left(u_{j}(0)+\ell_{j} \alpha_{j}\right) \\
& =\sum_{v_{i} \in k_{j} \ni v_{h} \neq v_{i}} u_{j}\left(v_{h}\right)+\nu_{i} \sum_{v_{i} \in k_{j}} \ell_{j}=\sum_{v_{i} \sim v_{h}} u_{i h}\left(v_{h}\right)+\nu_{i} \sum_{v_{i} \in k_{j}} \ell_{j},
\end{aligned}
$$

where we have used the adjacency setting. and $\mathbf{U}=\left(u_{s(i, h)}\right)_{i, h \in \mathcal{I}}$. Thus, we have shown

Lemma 8.1 A harmonic function under $(K C)$ satisfies

$$
\mathbf{U}(1) \mathbf{e}=\mathbf{U}(0)^{*} \mathbf{e}=-\left(\nu_{i} \delta_{i k}\right)_{\mathcal{I} \times \mathcal{I}} \mathcal{L}(\Gamma) \mathbf{e} .
$$

For $\nu=0$, in particular for non bipartite graphs, Lemma 8.1 is just equivalent with the defining relation for $\Pi_{\infty}^{+}(\Gamma)$. But in the bipartite case, it imposes restrictions. If all edge lengths amount to 1 e.g., then we have a very specific mean value property of the form

$$
\forall i \in \mathcal{I}: \nu_{i}=-\frac{1}{\gamma_{i}} \sum_{v_{i} \sim v_{h}} u_{i h}\left(v_{h}\right)
$$

\subsection{The unsigned incidence matrix in the finite case}

In view of applications in Section 5, we present some details for the unsigned incidence matrix $\mathcal{D}^{+}=\mathcal{D}^{+}(B)=\left(\left|d_{i j}\right|\right)_{n \times m}$ of a finite connected simple graph $B$ with $n$ vertices and $m$ edges. Recall that in this case

$$
\operatorname{dim} \Pi^{+}(B)=\operatorname{dim} \operatorname{ker} \mathcal{D}^{+}(B)= \begin{cases}m-n+1 & \text { if } B \text { is bipartite } \\ m-n & \text { if } B \text { is not bipartite. }\end{cases}
$$

As each edge is incident with exactly two vertices by simplicity, any $\varphi=\left(\varphi_{j}\right)_{m \times 1}$ fulfills

$$
\sum_{j=1}^{m} \varphi_{j}=\sum_{i=1}^{n} \sum_{d_{i j}=-1} \varphi_{j}=\sum_{i=1}^{n} \sum_{d_{i j}=1} \varphi_{j}
$$


In particular, if $B$ is bipartite and thereby endowable with the sink-source-orientation, (24) takes the form

$$
\sum_{v_{i} \text { sink }}\left(\mathcal{D}^{+}(B) \varphi\right)_{i}=\sum_{v_{i} \text { sink }} \sum_{v_{i} \in k_{j}} \varphi_{j}=\sum_{v_{i} \text { source }} \sum_{v_{i} \in k_{j}} \varphi_{j}=\sum_{v_{i} \text { source }}\left(\mathcal{D}^{+}(B) \varphi\right)_{i} .
$$

Thus, in the bipartite case, a vector of the canonical basis $\mathbf{e}_{i}^{n}$ of $\mathbb{R}^{n}$ can never belong to the image of $\mathcal{D}^{+}(B)$. This follows also from the fact that

$$
\forall j \in\{1, \ldots, m\}: \mathcal{D}^{+} \mathbf{e}_{j}^{m}=\mathbf{e}_{i}^{n}+\mathbf{e}_{k}^{n} \text { with } v_{i}, v_{k} \in k_{j}
$$

Finally, for application in Section 5, we can state the following

Lemma 8.2 Let $B$ be a finite bipartite graph, endowed with the sink-source orientation. Set

$$
\begin{aligned}
& s=\left(s_{i}\right)_{n \times 1}, \quad s^{+}=\left(s_{i}^{+}\right)_{n \times 1}, \quad s^{-}=\left(s_{i}^{-}\right)_{n \times 1}, \quad s_{i}=\sum_{v_{i} \in k_{j}} \ell_{j}, \\
& s_{i}^{+}=\left\{\begin{array}{ll}
s_{i} & \text { if } v_{i} \text { is a sink, } \\
0 & \text { otherwise, }
\end{array} \quad s_{i}^{-}= \begin{cases}s_{i} & \text { if } v_{i} \text { is a source, } \\
0 & \text { otherwise, }\end{cases} \right.
\end{aligned}
$$

Then

$$
s \in \operatorname{Im} \mathcal{D}^{+}(B), \quad \text { but } \quad s^{+}, s^{-} \notin \operatorname{Im} \mathcal{D}^{+}(B) .
$$

Proof. Clearly $\mathcal{D}^{+}$applied to the vector $\ell=\left(\ell_{j}\right)_{m \times 1}$ yields the vector $s=s^{-}+s^{+}$. Thus, it remains to show that $s^{+} \notin \operatorname{Im} \mathcal{D}^{+}$. But, if there were $\varphi$ with $\mathcal{D}^{+} \varphi=s^{+}$, (25) would lead to the contradiction

$$
L(B)=\sum_{v_{i} \text { sink }}\left(\mathcal{D}^{+}(B) \varphi\right)_{i}=\sum_{v_{i} \text { source }}\left(\mathcal{D}^{+}(B) \varphi\right)_{i}=0
$$

\section{Acknowledgments}

Joachim von Below is grateful to the research group GREDPA at UPC Barcelona for the invitation in 2018. José A. Lubary is grateful to the LMPA Joseph Liouville at ULCO in Calais for the invitation in 2018.

\section{References}

[1] Below, J. von, A characteristic equation associated with an eigenvalue problem on $C^{2}$-networks, Lin. Algebra Appl. 71 (1985) 309-325.

[2] Below, J. von, The index of a periodic graph, Results in Math. 25 (1994) 198223. 
[3] Below, J. von and Lubary, J.A., Harmonic functions on locally finite networks, Results in Math. 45 (2004) 1-20.

[4] Below, J. von and Lubary, J.A., The eigenvalues of the Laplacian on locally finite networks, Results in Math. 47 (2005) 199-225.

[5] Below, J. von, Lubary, J. A. and Vasseur, B., Some remarks on the eigenvalue multiplicities of the Laplacian on infinite locally finite trees, Results in Math. 63 (2013) 1331-1350.

[6] Below, J. von and Mugnolo, D., The spectrum of the Hilbert space valued second derivative with general self-adjoint boundary conditions, Linear Algebra and its Applications 439 (2013) 1792-1814

[7] Biggs, N. L., Algebraic graph theory. Cambridge Tracts Math. 67, Cambridge University Press, $1967^{1}, 1993^{2}$.

[8] Collatz, L., Spektren periodischer Graphen, Resultate der Mathematik 1 (1979) $42-53$.

[9] Lubary, J.A., Multiplicity of solutions of second order linear differential equations on networks, Lin. Alg. Appl. 274 (1998) 301-315.

[10] Lubary, J.A., On the geometric and algebraic multiplicities for eigenvalue problems on graphs, in: Partial Differential Equations on Multistructures, Lecture Notes in Pure and Applied Mathematics Vol. 219, Marcel Dekker Inc. New York, (2000) 135-146.

[11] Wilson, R. J., Introduction to graph theory, Oliver \& Boyd Edinburgh, 1972.

[12] Woess, W., Random walks on infinite graphs and groups, Cambridge Univ. Press 138, 2000.

Joachim von Below

LMPA Joseph Liouville ULCO, FR CNRS Math. 2956

Universités Lille Nord de France

50, rue F. Buisson, CS 80699, F-62228 Calais Cedex, France

email: below@univ-littoral.fr

phone 00.33.3.21.46.36.27

José A. Lubary

Departament de Matemàtiques

Universitat Politècnica de Catalunya, Campus Nord, Edifici $\Omega$

Jordi Girona, 1-3, 08034 Barcelona, Spain

email: jose.a.lubary@upc.edu 